\title{
Parity Violation in Chiral Molecules
}

\author{
Martin Quack ${ }^{\mathrm{a} *}$ and Jürgen Stohner ${ }^{\mathrm{a}, \mathrm{b}}$
}

Dedicated to Michele Parrinello on the occasion of his 60th birthday

\begin{abstract}
The current status of the computational approach to electroweak quantum chemistry which includes the parity violating weak nuclear interaction is briefly reviewed. After a short introduction and discussion of current experimental efforts in measuring parity violation in chiral molecules, the general theoretical background and recent approaches predicting order of magnitude increases in parity violating potentials are outlined. The current results on these increased values, the role of biomolecular chirality, calculation of properties, use of density functional theory, anharmonic vibrational and relativistic corrections, as well as a new isotope effect for isotopically chiral molecules are summarized.
\end{abstract}

Keywords: Biomolecular homochirality - Chiral molecules · Density functional theory · Electroweak quantum chemistry $\cdot$ Isotope effects $\cdot$ Parity violation

\section{Introduction}

Quantum theory has been serving our microscopic understanding of chemistry since the very beginning of quantum mechanics [1-10]. While the early work provided the conceptual background extending from molecular orbital theory [6-8][11] and valence bond theory [9] to the quantum mechanical foundations of stereochemistry, chirality, and tunneling [7][10], all of the early work had two major limitations. The first was of a technical nature as the necessary computations for the quantum mechanical many body problem arising in chemistry were too complex for the computational techniques available in the first half of the 20th century. This was already noted by Dirac in his fa-

${ }^{*}$ Correspondence: Prof. M. Quack ${ }^{a}$

${ }^{a} E T H$ Zürich (Hönggerberg)

Physical Chemistry, $\mathrm{HCl}$

$\mathrm{CH}-8093$ Zürich

Tel.: +4144632 4421

Fax: +4144632 1021

E-Mail: quack@ir.phys.chem.ethz.ch

bZürich University for Applied Sciences

Institute of Chemistry and Biotechnology ICB

Technikumstrasse 9

PO Box 805

$\mathrm{CH}-8401$ Winterthur

E-Mail: sth@zhwin.ch mous 1929 statement, expressing the belief that the theoretical basis for all of chemistry was available but that the computations were too complex to be carried out [5].

In the second half of the 20th century the advent of computational quantum chemistry removed many of the limitations mentioned by Dirac. Computational (quantum) chemistry started around 1950 following the enormous progress which was achieved in computer hardware and software development. The systematic progress in electronic structure calculations was honored by the 1998 Nobel Prize in chemistry to John D. Pople and Walter Kohn for their contributions to computational chemistry and physics [12][13].

Computational chemistry has proven to be useful to many branches of chemistry, ranging from applications to high-resolution molecular spectroscopy [14][15] in metal organic chemistry [16] and complex compounds [17] to condensed phase chemical dynamics [18], as well as biomolecular dynamics [19][20]. Indeed, a recent review issue in CHIMIA covers several of these now more standard aspects of computational chemistry [21]. It is probably fair to say that in spite of the enormous progress in useful applications to chemistry, all of the advances made essentially rely still on the foundations in quantum physics laid down by $1930[1-10]$.

The present short review deals with progress concerning the second much more fundamental conceptual limitation of early quantum physics as applied to chemistry. This limitation is related to a lack of under- standing and the corresponding omission in theory of certain fundamental interactions and symmetry violations, which are summarized now in the so-called electroweak theory of the Standard Model of Particle Physics (SMPP). Indeed, progress in computational chemistry over the last 40 years is somehow paralleled by the achievements in particle physics, culminating in the SMPP which has been developed in a similar time frame. In 1956 the parity non-conservation in particle decay was predicted theoretically [22] and experimentally discovered with only a short delay [23-25]. The left-handed helicity of neutrinos was measured in 1958 [26]. Zel'dovich [27] considered weak neutral currents and their influence on electronnucleon interactions in atoms. About ten years later, Glashow, Salam and Weinberg presented their 'electroweak model', unifying the electromagnetic and the weak force. Weak neutral currents were discovered at CERN in 1970, and after the discovery of the quark structure of hadrons, experimental results supported the SMPP with three quark and lepton families. Two charged $\left(\mathrm{W}^{+}, \mathrm{W}^{-}\right)$ and a neutral $\left(\mathrm{Z}^{0}\right)$ heavy boson which mediate the electroweak interaction are predicted within the SMPP. These bosons were discovered in 1983 and precise measurements of their masses and lifetimes were carried out at CERN (LEP) and Stanford (LCP); the results supported the SMPP and allowed also the determination of the number of particle families (three) within this framework [28][29]. Renormalization has rendered electroweak theory a quantitatively useful tool for many areas of physics [30][31]. 
Not very long after the discovery of parity violation in nuclear physics, it was realized that it might introduce a tiny energy difference between enantiomers, left- vs right-handed molecules of a chiral compound [32][33]. This has possible consequences for homochirality in nature, i.e. nature's almost exclusive preference of L-amino-acids over D-amino-acids and D-sugars over L-sugars in the biopolymers of the chemistry of life [32-43]. The specific role of parity violation in this context is, however, still subject to debate (see [40] and references therein).

Quantitative computations of parity violation in chiral molecules go back to the early work of Hegström, Rein and Sandars [44][45] and Mason and Tranter [46][47]. It was particularly the conclusions from these early calculations showing supposedly that parity violation induces a larger systematic stability of the natural L-amino acids and D-sugars, which received considerable attention and publicity in the 1980s and early 1990s. A dramatic change occurred, however, when we showed about a decade ago with improved theoretical methods that for the benchmark molecules $\mathrm{H}_{2} \mathrm{O}_{2}$ and $\mathrm{H}_{2} \mathrm{~S}_{2}$ parity violating energy differences between enantiomers are increased by about two orders of magnitude (see Table 2 in Section 4.1. [35-38]). One often finds similarly large increases for other chiral molecules. Furthermore, we could show by general arguments and specific calculations that the earlier conclusions on the relative stability of L- and D-amino acids could not be maintained [35-38] (see Section 4.2.). These findings have obvious consequences for doing experiments on parity violation, which look much more promising today than 20 years ago with the now much increased predicted effects. They also change our outlook on their potential importance in biochemical selection of chirality in early evolution.

Fig. 1 shows that with a slight time lag after this discovery, there was a considerable increase in publication activity on molecular parity violation. In order to appreciate the causal relationship one must, of course, look into the publications in more detail. It is not the place here to go into the finer historical details. It should suffice to state that presumably all groups active in the field at the time of the discovery and most of those entering later might have been aware of the striking results of the 1995/96 work [35] and were probably influenced by it [48]. In any case, the sequence of events is unquestioned.

After these remarks on new developments in the field of molecular parity violation, we will, in Section 2, provide a brief review of current experimental concepts and efforts, and in Section 3 some of the current theoretical background as well as

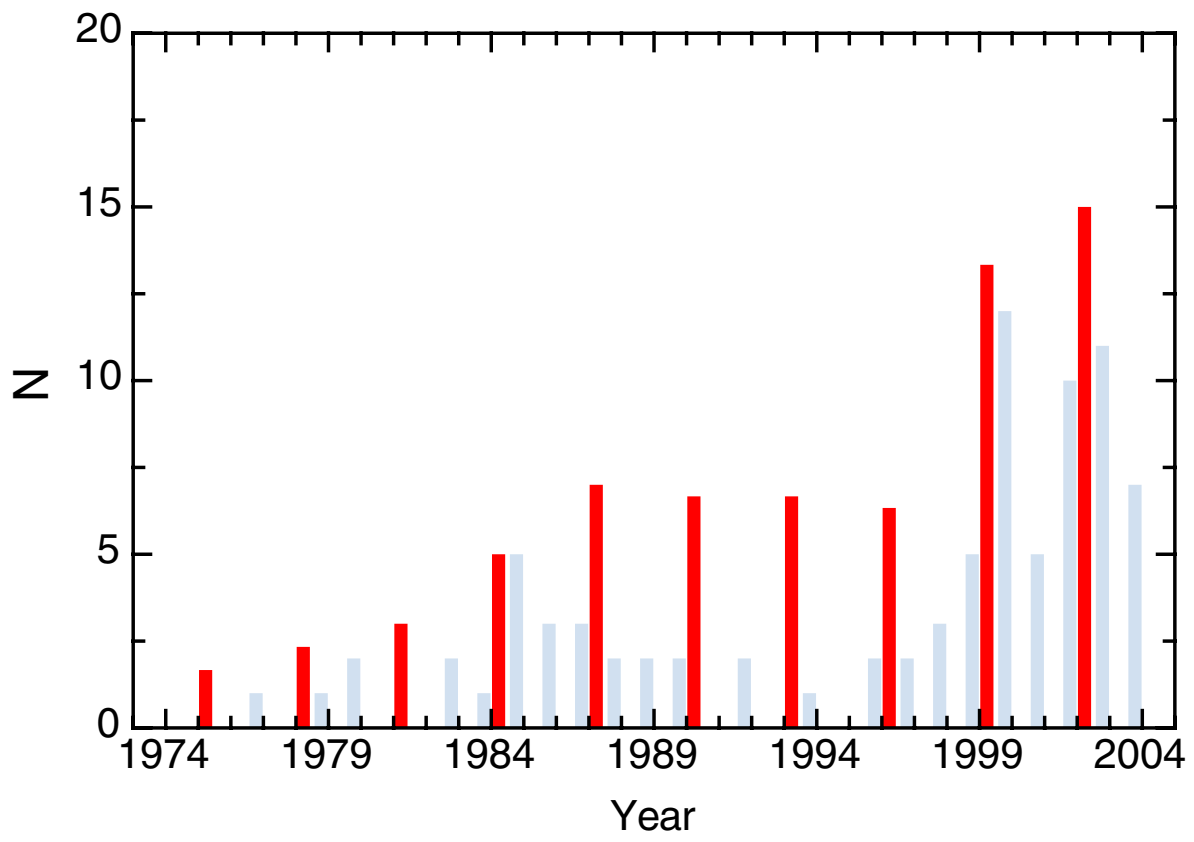

Fig. 1. Number of publications between 1974 and 2004 that deal with parity violation in molecules. Prior to 1974 the number of such papers is very small, indeed. Starting from 1975, there appears the average number of papers that deal with parity violation shown in intervals of three years (dark, red bars; the 1975 value displayed is obtained from the years 1974, 1975, and 1976, etc.). The lighter (blue) bar shows the total non-averaged number of papers that calculate parity violating effects (energy difference, frequency shift, etc., these are also included in the averaged data). Not shown are those papers which just try to relate parity violation to some macroscopic (mostly thermodynamic) quantity in some general discussions or experiments (see text for details). The data for 2004 are presumably not yet complete. the current status of computations on molecular parity violation in chiral molecules and beyond.

\section{Experimental Approaches}

We shall review briefly only the main concepts of current experimental approach- es as far as they are relevant to the understanding of current theoretical efforts concerning the prediction of the experimental effects.

Fig. 2 outlines the basic features of spectroscopic schemes on molecular parity violation in the gas phase as currently pursued in a few laboratories. The first proposal historically concerned the measure-

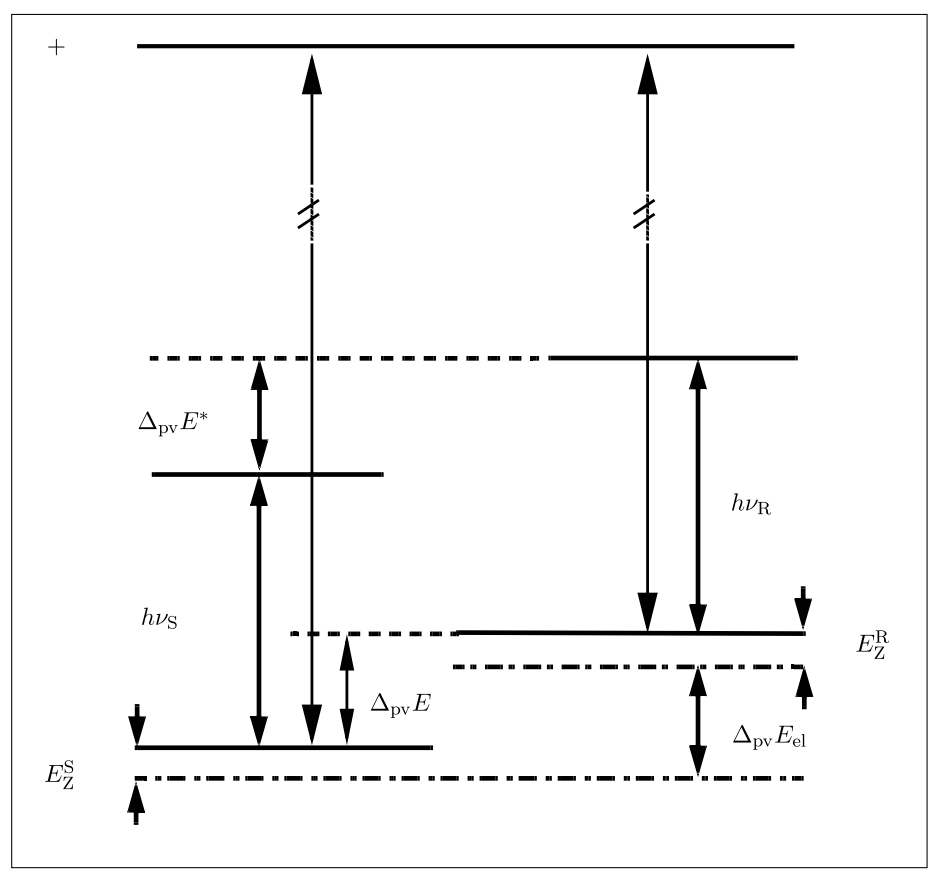

Fig. 2. Energy level scheme to illustrate observable spectroscopic transitions in enantiomers of chiral molecules, see text for details 
ment of shifts of spectroscopic transition frequencies in an $(S)$-enantiomer compared to those in an $(R)$-enantiomer, thus the measurement of $h v_{\mathrm{S}}$ and $h v_{\mathrm{R}}$ in Fig. 2. Without parity violation one would have $h v_{\mathrm{S}}=h v_{\mathrm{R}}$ by symmetry. With parity violation, one can define a relative parity violating frequency shift

$$
\frac{\Delta_{\mathrm{pv}} v}{v}=\frac{v_{\mathrm{R}}-v_{\mathrm{S}}}{v_{\mathrm{R}}}
$$

The proposal to search for such shifts in infrared spectroscopy goes back 30 years [49] and since that time a number of proposals were made and some experiments were carried out in the infrared [49-53], microwave [53] and $\gamma$-ray (Mössbauer) spectral ranges [54]. Experiments reached accuracies $\Delta v / v$ from $10^{-6}$ in 1976 to $10^{-14}$ recently [51][52], but in no case molecular parity violation could be demonstrated, so far. The prediction for $\mathrm{CHBrClF}$, which was investigated repeatedly, is about $\Delta v / v=$ $10^{-16}$ in the infrared [55-62] and the negative experimental results thus are consistent with theory. It may be noted that this experimental concept does not allow one to determine the energy difference $\Delta_{\mathrm{pv}} E$ between enantiomers (see Fig. 2) rather one would obtain the difference of such differences, namely $\Delta_{\mathrm{pv}} E^{*}-\Delta_{\mathrm{pv}} E$.

Another type of experiment allows one to directly determine the energy difference
$\Delta_{\mathrm{pv}} E$, which corresponds also to the heat of reaction of the stereomutation process

$$
\mathrm{R}=\mathrm{S} \quad \Delta_{\mathrm{r}} H_{0}^{0}=\Delta_{\mathrm{pv}} H_{0}^{0}=N_{\mathrm{A}} \Delta_{\mathrm{pv}} E
$$

The idea behind this second scheme is to use transitions to an achiral intermediate excited state with rovibronic levels of well-defined parity (e.g. the plus sign for positive parity in Fig. 2). This then provides by combination difference from the $\mathrm{R} \leftrightarrow+$ and $\mathrm{S} \leftrightarrow+$ the difference $\Delta_{\mathrm{pv}} E$. As discussed in some detail elsewhere, the experiment can be carried out in the frequency or time domain [34][63], and this type of approach is currently pursued in the Zürich group. A necessary condition for $\Delta_{\mathrm{pv}} E$ being an experimentally measurable quantity as referred to in Eqn. (2) is, however, that it is much larger than the tunneling splitting $\Delta_{ \pm} E$ for the hypothetical symmetrical potential without parity violation, thus

$$
\Delta_{\mathrm{pv}} E \gg \Delta_{ \pm} E
$$

This condition is generally met for chiral molecules that are stable for days or longer as discussed in [40].

To conclude the discussion of the experimental concepts we might note here that it has also been proposed to measure time-dependent optical activity in chiral molecules with the special property $\Delta_{\mathrm{pv}} E \approx \Delta_{ \pm} E$, where the amplitude of the oscillation depends upon the relative magnitude of $\Delta_{\mathrm{pv}} E$ and $\Delta_{ \pm} E$ [64][65]. To our knowledge no ongoing experimental efforts exist to date along these lines, although in our calculations we have identified some molecules where the conditions for such experiments might be met in principle [66-68], in contrast to high barrier cases [40][55-62][69].

\section{Theory}

\subsection{The Standard Model of Particle Physics (SMPP)}

Quite generally, a standard model can be understood as a theoretical framework which is based on experimental observations and which allows new data to be predicted [70]. In this respect, the periodic system of elements can also be viewed as a standard model which is common to every chemist. The use of the notion 'model' arises from the fact that the theoretical framework leaves open a number of parameters, which have to be determined experimentally (such as the charge and the mass of the electron in the SMPP). The main achievement of the SMPP was the unification of the weak and the electromagnetic forces and to some extent the strong force in a quantum gaugefield theoretical language.

Table 1 summarizes our current knowledge of interactions and elementary particles of quarks and leptons as they appear

Table 1. Summary of currently known elementary (point-like) particles with their approximate masses (top) and their interactions [28][71]. The essence of experimental data from high energy physics can be accounted for by these entries. To each particle one has an antiparticle of opposite charge (not listed here [29]).

STANDARD MODEL OF PARTICLE PHYSICS: PARTICLES

LEPTONS $(S=\hbar / 2$, Fermions)

Mass $m /\left(\mathrm{GeVc}^{-2}\right)$

Mass $m /\left(\mathrm{GeVc}^{-2}\right)$

QUARKS ( $S=\hbar / 2$, Fermions)

Mass $m /\left(\mathrm{GeVc}^{-2}\right)$

Mass $m /\left(\mathrm{GeVc}^{-2}\right)$

Force:

\section{Strong}

SU(3)

Relative Strength

Range

Gauge Bosons $(S=1 \hbar)$

Mass $m /\left(\mathrm{GeVc}^{-2}\right)$

Particles $<10^{-2}$

$$
\begin{gathered}
v_{\mathrm{e}} \\
<2.2 \cdot 10^{-9} \\
\mathrm{e}^{-} \\
5 \cdot 10^{-4} \\
\\
u_{1}, u_{2}, u_{3} \\
5 \cdot 10^{-3} \\
d_{1}, d_{2}, d_{3} \\
10^{-2}
\end{gathered}
$$

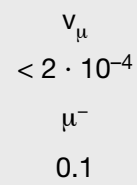

$$
c_{1}, c_{2}, c_{3}
$$$$
1.3
$$$$
s_{1}, s_{2}, s_{3}
$$

0.2

$$
\begin{gathered}
\mathrm{v}_{\tau} \\
<2 \cdot 10^{-2} \\
\tau^{-} \\
1.8
\end{gathered}
$$

$$
t_{1}, t_{2}, t_{3}
$$$$
174
$$$$
b_{1}, b_{2}, b_{3}
$$

4.3

\section{INTERACTIONS AND FIELD PARTICLES}

$$
\text { Electromagnetic }
$$

$S U(2) \otimes U(1)$

$$
1 / 137
$$

\section{1 to $1 \mathrm{fm}$}

Gluons $g_{1} \ldots g_{8}$

Hadrons
Photon $\gamma$

$<10^{-24}$

charged
Weak

$\approx 10^{-5}$

$<0.1 \mathrm{fm}$

$W^{ \pm}, Z^{0}$

$\approx 80, \approx 91$

Hadrons, Leptons

$$
Q=0 \text { e }
$$$$
Q=-1 \mathrm{e}
$$$$
\text { Gravitation }
$$$$
\approx 10^{-38}
$$

$(\infty)$

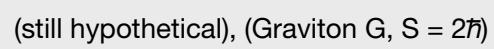


in the SMPP [28][71]. As every chemical compound can be decomposed in its elements, every chemical element is ultimately made-up of quarks and leptons. They have spin of $(1 / 2) \hbar=(1 / 2) h /(2 \pi)(h$ is Planck's constant) and are therefore fermions. The electron, muon, and tauon $\left(e^{-}, \mu^{-}, \tau\right)$ carry charge of $-1 \mathrm{e}$ ( $\mathrm{e}$ is the elementary charge) and a mass which is listed as $\mathrm{GeV}^{-2}$ (c is the velocity of light in vacuo) following Einstein's mass/energy relationship $m$ $=E / c^{2}$. The corresponding neutrinos $v_{i}(i$ $=e, \mu, \tau)$ are electrically neutral and have much lower masses. The quarks also carry spin $(1 / 2) \hbar$ and are fermions. The up (u), charm $(\mathrm{c})$, and top $(\mathrm{t})$ quark carry a charge of $(2 / 3)$ e whereas the down (d), strange (s), and bottom (b) quark carry a charge of -(1/3)e. All quarks come in three colors distinguished by the indices 1,2 , and 3 . To every elementary particle, there exists a corresponding anti-particle which has the same spin and mass (within CPT symmetry) but opposite electric charge. It is common to collect the elementary particles in families: the first family (second column in Table 1) contains the electron, the electron neutrino, the up- and the down-quarks. They constitute the nucleons, the pions, and other mesons which are responsible for nuclear binding; the electron (positron) and the electron (anti)neutrino belong to this family and they appear in nuclear $\beta$-decay. The other families consist of much heavier and rather short-lived particles. The strong force (quantum chromo-dynamics, QCD) between colored quarks is mediated by spin-1 bosons (gluons, g) which themselves carry color charge. The interaction between quarks and gluons increase with distance and therefore, free quarks and gluons cannot exist. Nucleons consist of three colored quarks with 'white' net-color (color neutral). The short range of the strong force is characterized by the Compton wavelength of the lightest $\pi$ meson. The electromagnetic force is mediated by the photon (spin1 boson), which is massless and therefore accounts for the long range of the electromagnetic interaction between charged particles. The very short range weak force is mediated by the neutral $\mathrm{Z}$ and the charged $\mathrm{W}$ bosons. Within the SMPP as developed by Weinberg, Salam and Glashow in the late $60 \mathrm{~s}$ and renormalized by t'Hooft and Veltman (for a review, see [70] and references cited therein), the interaction energy between leptons and quarks can be written as [71]

$L=g \boldsymbol{J}_{\mu} \cdot \boldsymbol{W}^{\mu}+g^{\prime \prime} J_{\mu}^{\mathrm{Y}} B^{\mu}$

$J_{\mu}$ is the weak iso-spin current, $J_{\mu}^{Y}$ the weak hyper-charge current $\left(Y=Q-I_{3}, Q\right.$ is the electric charge and $I_{3}$ the third com- ponent of the weak iso-spin), and $\boldsymbol{W}^{\mu}, B^{\mu}$ are the 4-potentials associated with the boson fields. $g$ and $g$ " are coupling constants which are related to the Weinberg angle $\theta_{\mathrm{W}}$ as $g / g^{\prime \prime}=\tan \theta_{\mathrm{W}}$. Inserting the tensor components of the weak currents (with the boson fields of the charged particles $\mathrm{W}^{ \pm}$the neutral $\mathrm{Z}$, and the photon $\mathrm{A}$ ) leads to the Lagrange density $L$

$$
\begin{aligned}
L_{\mathrm{SMPP}}= & \frac{g}{\sqrt{2}}\left(J_{\mu}^{-} W^{\mu+}+J_{\mu}^{+} W^{\mu-}\right)+ \\
& \frac{g}{\cos \theta_{W}}\left(J_{\mu}^{3}-\sin ^{2} \theta_{W} J_{\mu}^{\mathrm{em}}\right) Z^{\mu}+ \\
& g \sin \theta_{\mathrm{W}} J_{\mu}^{\mathrm{em}} A^{\mu}
\end{aligned}
$$

$$
=L_{\mathrm{CC}}+L_{\mathrm{NC}}+L_{\mathrm{EM}}
$$

where the indices denote weak charged current (CC), weak neutral current (NC) and electromagnetic neutral current (EM). The latter neutral current gives the elementary electric charge $e=g \sin \theta_{\mathrm{W}}$. The Fermi constant is $G_{\mathrm{F}}=\sqrt{2} g^{2} /\left(8 M_{\mathrm{W}}^{2}\right)$ where $M_{\mathrm{W}}$ denotes the mass of $\mathrm{W}^{ \pm}\left(M_{\mathrm{W}} \approx(37.4)\right.$ $\left.\left.\sin \theta_{\mathrm{W}}\right) \mathrm{GeV} \approx M_{\mathrm{z}} \cos \theta_{\mathrm{W}}\right)$. Starting out from these general expressions for the relativistic currents the path, which leads from the relativistic approach to a set of practical expressions, involves in essence the following approximations or neglects: (i) taking the low energy semi-relativistic limit; (ii) neglecting the electron-electron parity violating interaction; (iii) using the Breit-Pauli form for the semi-relativistic magnetic (one- and two-electron spin-orbit) interactions and (iv) representing the nucleus $a$ by a pointlike object with the electric charge $Z_{a}$ e and electroweak charge (neglecting radiative corrections)

$$
Q_{a} \approx\left(1-4 \sin ^{2}\left(\theta_{\mathrm{W}}\right)\right) \cdot Z_{a}-N_{a}
$$

where $N_{a}$ is the neutron number of nucleus $a ; Z_{a}$ the nuclear charge number, and the Weinberg angle with $\sin ^{2} \theta_{\mathrm{W}} \approx 0.2319$ [72] We note that details of these approximations have been discussed in a number of places [36][37][45][46][73-76][79] and an alternative approach has been formulated that leads to the Dirac-Fock theory of molecular parity violation [61][77]]. In addition to the critical discussion in [36] we draw attention to an extensive review of the theory presented in [75]. We note that all of the approximations might be removed or can be checked when this appears necessary.

\subsection{Electroweak Quantum}

Chemistry: Parity Violating Potential

The approximations discussed above lead to a practical set of quantum chemi- cal equations allowing one to calculate for each configuration of nuclei in a molecule a parity violating potential (in addition to the usual electrostatic or electromagnetic potentials) and for this type of quantum chemistry we coined the name 'electroweak quantum chemistry' [35][36]. In a semi-relativistic approximation and in the absence of external electromagnetic fields, two contributions for each electron $i$ may be considered relevant in order to compute the parity violating interaction $\hat{H}_{\mathrm{pv}}^{\mathrm{eN}}$, namely [36][74][78][79]:

$$
\hat{H}_{\mathrm{pv}}^{\mathrm{eN}}=\frac{G_{\mathrm{F}}}{2 m_{\mathrm{e}} c \sqrt{2}}\left(\hat{H}_{\mathrm{pv}}^{(1)}+\hat{H}_{\mathrm{pv}}^{(2)}\right)
$$

with $m_{\mathrm{e}}$ : electron mass; $c$ : speed of light; $G_{\mathrm{F}}$ $\approx 1.43586 \times 10^{-62} \mathrm{Jm}^{3}$ : Fermi weak coupling constant which is derived from the muon lifetime $\tau_{\mu}$ [80]. The individual terms are as follows:

$$
\hat{H}_{\mathrm{pv}}^{(1)}=Q_{\mathrm{a}} \overrightarrow{\boldsymbol{s}} \cdot[\overrightarrow{\boldsymbol{p}} \delta(\overrightarrow{\boldsymbol{r}}-\overrightarrow{\boldsymbol{r}}(a))+\delta(\overrightarrow{\boldsymbol{r}}-\overrightarrow{\boldsymbol{r}}(a)) \overrightarrow{\boldsymbol{p}}]
$$

$$
\begin{aligned}
& \hat{H}_{\mathrm{pv}}^{(2)}=-4 \lambda_{\mathrm{a}}\left(1-4 \sin ^{2}\left(\theta_{W}\right)\right) \\
& (\overrightarrow{\boldsymbol{s}} \cdot[\overrightarrow{\boldsymbol{p}} \delta(\overrightarrow{\boldsymbol{r}}-\overrightarrow{\boldsymbol{r}}(a))+\delta(\overrightarrow{\boldsymbol{r}}-\overrightarrow{\boldsymbol{r}}(a)) \overrightarrow{\boldsymbol{p}}])(\overrightarrow{\boldsymbol{s}} \cdot \overrightarrow{\boldsymbol{I}}(a))
\end{aligned}
$$

with $\overrightarrow{\boldsymbol{s}}$ : reduced dimensionless electron spin, $\overrightarrow{\boldsymbol{p}}$ : electron linear momentum, e: elementary charge, $\delta(\overrightarrow{\boldsymbol{r}}-\overrightarrow{\boldsymbol{r}}(a))$ : three dimensional Dirac delta distribution confining the interaction locally to the (point) nuclear position, $\overrightarrow{\boldsymbol{r}}(a)$ : position vector of the nucleus. $\lambda_{\mathrm{a}}$ is a nuclear parameter close to 1 [36][74-76][79]. $\hat{H}_{\mathrm{pv}}^{(2)}$ depends on the reduced dimensionless nuclear spin $\vec{I}(a)$. In atomic and molecular physics, however, this nuclear spin dependent part is considered relatively small and therefore usually neglected which then leads to $\hat{H}_{\mathrm{pv}}^{\mathrm{eN}} \approx G_{\mathrm{F}} /\left(m_{e} c 2 \sqrt{2}\right) \hat{H}_{\mathrm{pv}}^{(1)}$. Nuclear spin dependent effects have therefore been neglected in calculations of molecular parity violation except in cases where one is specifically interested in observables that depend on nuclear spin, as for example the NMR chemical shift [74][78][81][82]. The nuclear spin dependent term in $\hat{H}_{\mathrm{pv}}^{(2)}$ is weighted by a factor $\left(1-4 \sin ^{2} \theta_{\mathrm{W}}\right) \approx 0.07$ which is rather small compared to the weighting with $Q_{a}$ in Eqn. (9) which grows roughly proportional to the number of neutrons $N$ [36][45]. Thus, we might neglect in a first approximation the nuclear spin dependent term in the molecular parity violating Hamiltonian which itself is obtained from the atomic Hamiltonian by a simple sum over all nuclei $a$ and electrons $i$, as 
discussed by [36][45][46] and reads (see $[36][45][46][74][78][79][81]$ and references therein)

$$
\begin{aligned}
& \hat{H}_{\mathrm{pv}} \approx \frac{G_{\mathrm{F}}}{2 m_{\mathrm{e}} c \sqrt{2}} \sum Q_{a} \sum \overrightarrow{\boldsymbol{s}}(i) . \\
& {[\overrightarrow{\boldsymbol{p}}(i) \delta(\overrightarrow{\boldsymbol{r}}(i)-\overrightarrow{\boldsymbol{r}}(a))+\delta(\overrightarrow{\boldsymbol{r}}(i)-\overrightarrow{\boldsymbol{r}}(a)) \overrightarrow{\boldsymbol{p}}(i)]}
\end{aligned}
$$

Eqn. (11) has been used in the nonrelativistic (or rather semi-relativistic) limit to calculate parity violation in molecules in absence of an external magnetic field and upon neglecting nuclear spin effects [36][44-47][79]. The corresponding expression derived from the relativistic Dirac equation would be [77][83][84]

$$
\hat{H}_{\mathrm{pv}}^{\mathrm{rel}} \approx \frac{G_{\mathrm{F}}}{2 \sqrt{2}} \sum_{a} Q_{a} \sum \gamma_{\mathrm{i}}^{5} \rho_{a}(\vec{r}(i))
$$

or similarly from a relativistically parameterized extended Hückel method [74] In Eqn. (12), $\rho_{a}(\vec{r}(i))$ represents the (nonpoint-like) normalized nucleon density. The pseudo scalar is given by

$$
\gamma^{5}=\gamma_{5}=\left(\begin{array}{ll}
0 & I \\
I & 0
\end{array}\right)
$$

with the $2 \times 2$ unit matrices $I$.

An estimate of the parity violating potential $V_{\mathrm{pv}}(q)$ resulting from an interaction between an electron $i$ and a nucleus $a$ has first been obtained from a perturbation theoretical sum-over-states (SOS) evaluation of the electronic ground state expectation value of the parity-violating Hamilton operator, Eqn. (11) [35][36][45][46][73][74] [85][86] from

$$
V_{\mathrm{pv}}(q)=2 \cdot \operatorname{Re} \sum_{n} \frac{\left\langle 0\left|\hat{H}_{\mathrm{so}}\right| n\right\rangle \cdot\left\langle n\left|\hat{H}_{\mathrm{pv}}\right| 0\right\rangle}{E_{0}-E_{n}}
$$

The real part of the sum-over-states expression gives $V_{\mathrm{pv}}$ at each geometry characterized by a set of suitable coordinates. The evaluation of the sum in Eqn. (14) is numerically demanding due to convergence problems with respect to the number of basis functions and the number of electronically excited triplet states $n$ with energy $E_{n}$ entering the SOS expression. The expectation value of the parity violation operator for ground state singlet wave-functions is exactly zero since $\hat{H}_{\mathrm{pv}}$ is a triplet operator. In the non-relativistic approach, some excited state triplet character must be present. This is achieved by the contribution of the spinorbit interaction $\hat{H}_{\mathrm{SO}}$, Eqn. (14). Various degrees of approximation have been used for $\hat{H}_{\mathrm{SO}}$, namely representing $\hat{H}_{\mathrm{SO}}$ by the one-electron operator part using empirical spin-orbit parameters or from exact (BreitPauli)expressions [35][36][45][46][73][74] [79][85] and combined with the two-electron part [73][79]. The matrix elements of the operators $\hat{O}\left(\hat{H}_{\mathrm{SO}}\right.$ and $\left.\hat{H}_{\mathrm{pv}}\right)$ evaluated in a Gaussian-type orbital atomic basis set $\chi$ have been transformed to the molecular orbital basis set $\phi$ with a transformation matrix $\mathbf{C}$ from a separate $a b$ initio calculation according to [35][36][86]

$$
|\phi\rangle=C^{\dagger}|\chi\rangle
$$

$$
\langle\phi|\hat{\mathbf{O}}| \phi\rangle=\left\langle\chi\left|\mathbf{C} \cdot \hat{\mathbf{O}} \cdot \mathbf{C}^{\dagger}\right| \chi\right\rangle
$$

When the large increase of parity violating potentials within a CIS (configuration interaction with singles excitation) framework was found [35][36], compared to previously accepted results [44-47], we pursued the following strategy to secure these results. We developed a completely independent computer program based on the sum-over-states expression but allowing for a more efficient incorporation of various quantum chemical electronic wave function types [86]. Secondly, as the sumover-states expression showed very slow convergence particularly for larger molecules, we circumvented its use altogether in a multi-configuration linear response (MCLR) approach. In the framework of propagator techniques, the parity violating potential in Eqn. (14) in particular can be represented by an expression from response theory [79]

$$
V_{\mathrm{pv}}=\left\langle\left\langle\hat{H}_{\mathrm{pv}} ; \hat{H}_{\mathrm{so}}\right\rangle\right\rangle_{\omega=0}=\left\langle\left\langle\hat{H}_{\mathrm{so}} ; \hat{H}_{\mathrm{pv}}\right\rangle\right\rangle_{\omega=0}
$$

General information on the propagator technique can be found in [79][87][88] (and refs. therein). One can say that the parity violating potential is the response of $\left\langle\Psi_{0}\left|\hat{H}_{\mathrm{px}}\right| \Psi_{0}\right\rangle$ to the static $(\omega=0)$ perturbation $H_{\mathrm{SO}}$ or vice versa. This approach shows good convergence and frequently the random phase approximation (RPA) is found a sufficiently good (but not necessary) approximation for the ground state wavefunction $\Psi_{0}$.

\section{Short Review of Some Results and Main Conclusions Drawn from Current Work}

\subsection{Increase of Parity Violating Potentials by Order of Magnitude}

The first recent findings to be discussed concerns the large increase of parity violating potentials found in our 1995/6 work. As pointed out in considerable detail in [36], the previously used and accepted single determinant excitation restricted HartreeFock (SDE-RHF) is particularly deficient with respect to the description of the excited state wavefunctions and energies appearing in Eqn. (14). The simplest improvement by using a configuration interactions with singles excitation (CIS-RHF) approach led to very large increases of parity violating potentials for the benchmark molecules $\mathrm{H}_{2} \mathrm{~S}_{2}$ and $\mathrm{H}_{2} \mathrm{O}_{2}$. As discussed in Section 3 these results were confirmed by further developments in our own group and later independently by other groups as summarized in an exemplary fashion in Table 2. From the results summarized here, it would appear that this large increase can be considered to be well established. $E_{\mathrm{pv}}$ without nuclear spin [35][36][94] and with nuclear spin [74] can be formulated as a trace of a tensor. As discussed in [35] because of the possible numerical analysis of $E_{\mathrm{pv}}$ in the early calculations as a trace of a tensor, which is thus the sum of three partly compensating terms with possibly different signs, all results prior to 1995 on the basis of SDE-RHF and related approaches must be considered not only refuted with respect to magnitude but also cannot be relied on for sign even though the sign sometimes may agree, but sometimes not. The latter especially concerns the calculation of parity violating effects in biomolecules.

\subsection{Biomolecular Homochirality and Molecular Parity Violation}

Parity violating energies have been calculated for zwitter ionic forms of amino acids in the past [35][36][46][73][85][95] [96] (and refs. cited therein). As discussed in Section 4.1 because of the large errors of the early calculations all the results and conclusions drawn on the apparent stabilization of the natural L-amino acids and D-sugars obtained and published prior to 1995/6 (see [97][98] and refs. cited therein) had to be questioned [35-38]. In two independent careful investigations of parity violation in alanine published back to back in 2000 it was demonstrated that the earlier results on this molecule could not be maintained, neither in the gas phase nor in solution [38][99]. It was also pointed out that biochemical precursor molecules and transition states should be calculated [39]. The calculation of parity-violating effects on alanine by explicitly considering the water environment on the basis of a Monte Carlo simulation [100] came to the same conclusions as obtained by representing the solute by a continuum model [38][99]. Two critical reviews of the current situation come to the conclusion that the question of relevance of parity violation for the selection of biomolecular homochirality must be considered completely open [40][101]. 
Table 2. Benchmark calculations on parity violating energy differences in $\mathrm{H}_{2} \mathrm{O}_{2}$ and $\mathrm{H}_{2} \mathrm{~S}_{2}$ with various ab initio methods: TDA (Tamm-Damcoff approximation), MC-LR (Multi-configurational linear-response), DHF (Dirac-Hartree-Fock), Rel-MBPT2 (relativistic many-body perturbation theory second order), Rel-CCSD(T) (relativistic coupled cluster singles, doubles and perturbative triples excitation), DFT (density functional theory), ZORA (zeroth order regular approximation). The values $E_{\mathrm{pv}} / 10^{-20} E_{\mathrm{h}}$ are for a dihedral angle of 45 degrees (or arithmetic mean for 30 and 60 degrees when no value available). $\left|\Delta_{\mathrm{pv}} E\right|$ is taken at the equilibrium structure.

\begin{tabular}{|c|c|c|c|c|}
\hline Molecule & Method & $\operatorname{Ref}(\mathbf{s})$ & $\begin{array}{l}N_{\mathrm{A}} \cdot\left|\Delta_{\mathrm{pv}} E\right| \\
/ 10^{-12}{\mathrm{~J} \mathrm{~mol}^{-1}}^{-1}\end{array}$ & $E_{\mathrm{pv}} / 10^{-20} E_{\mathrm{h}}$ \\
\hline \multirow{9}{*}{$\mathrm{H}_{2} \mathrm{O}_{2}$} & SDE-RHF(6-31G) & [40], [46] & 0.0036 & -1.2 \\
\hline & CIS-RHF (6-31G) & [35-37], [40], [94] & 0.60 & -39.7 \\
\hline & TDA (6-31G) & [89] & 0.84 & -55.9 \\
\hline & CASSCF-LR (cc-pVTZ) & [79] & 0.41 & -33.4 \\
\hline & DHF & [77] & 0.45 & -71.6 \\
\hline & Rel-MBPT2; Rel-CCSD(T) & [90] & & $-58.6 ;-62.0$ \\
\hline & Rel-ZORA & [91] & 0.47 & -79.3 \\
\hline & Rel-DFT ZORA & [92] & 0.99 & -69.3 \\
\hline & Dirac-Coulomb MP2 & [93] & 0.88 & -57.9 \\
\hline \multirow{9}{*}{$\mathrm{H}_{2} \mathrm{~S}_{2}$} & SDE-RHF(4-4-31G) & [40], [46] & 0.24 & -135.0 \\
\hline & CIS-RHF (6-31G) & [35-37], [40], [94] & 22.5 & -1654.2 \\
\hline & TDA (4-4-31G) & [89] & 19.2 & -1487.7 \\
\hline & $\begin{array}{l}\text { MC-LR RPA } \\
\text { (aug-cc-pVTZ) }\end{array}$ & [66], [94] & 22.4 & \\
\hline & DHF & {$[77]$} & 33.5 & -2263.6 \\
\hline & Rel-MBPT2; Rel-CCSD(T) & [90] & & $-2146.0 ;-2140.8$ \\
\hline & Rel-ZORA & [91] & 35.2 & -2346.0 \\
\hline & Rel-DFT ZORA & [92] & 34.7 & -2692.0 \\
\hline & Dirac-Coulomb MP2 & [93] & 26.8 & -2112.0 \\
\hline
\end{tabular}

[110]. The results of our first calculation are reproduced in Fig. 3 together with HF and RPA data.

At that time, spin-orbit interaction was not part of deMon and we had to evaluate $V_{\text {pv }}$ based on Eqn. (14) using the KohnSham orbitals to perform the transformation according to Eqns. (15) and (16). Depending on the functionals used, there is a change of sign in $V_{\text {pr }}$ between $\mathrm{HF}$ and DFT or RPA and DFT (using standard local spin density (LSD) exchange functional combined with the Vosko, Wilk, Nusair (VWN) parametrization of the correlation functional) as far as the equilibrium value at $q=0$ is concerned. This is a major drawback because the sign of $V_{\mathrm{pv}}$ at equilibrium is relevant for the relative stability of the enantiomers. This problem is still unsolved even today as can be seen in a recent DFT calculation [93] (Fig. 1). It seems that DFT is currently much more volatile than $a b$ initio calculations, as sign changes in $V_{\mathrm{pv}}$ (e.g. caused by different $a b$ initio methods or basis sets employed) have not been observed for molecules at higher levels of theory. This, however, has little effect on the determination of frequency shifts as they depend on the derivative with respect to $q$. $V_{\mathrm{pv}}$ as a function of $q$ as obtained from DFT is only shifted which results in minor changes in the slope. Results for $\mathrm{H}_{2} \mathrm{O}_{2}$ turn out to be rather acceptable [111]. Density functional theory should become useful for the calculation of parity violation in larger molecules, however, it is presumably less

\subsection{Calculation of Properties}

Table 3 summarizes some calculations of properties (heat of reaction or stereomutation $\Delta_{\mathrm{r}} H_{0}^{0}$, relative frequency shifts $\Delta_{\mathrm{pv}} v / v$, relative changes in rotational constants $\Delta_{\mathrm{pv}} X /$ $X$, specific heat at constant volume, $\mathrm{C}_{\mathrm{v}}$, or the equilibrium constant $K_{\mathrm{p}}$ ) related to parity violation in chiral molecules. We give these current results which might turn out to be important in the infrared and microwave spectral ranges here only as examples of the many possible calculated properties which are influenced by parity violation.

\subsection{Use of Density Functional Theory}

An extension of calculations of parity violation to larger molecules suggests the use of density functional theory. During an academic visit of one of us (JS) at the Université de Montréal in the group of Denis Salahub in 2000 , we calculated the parity violating potential as a function of the $\mathrm{CF}$ stretching normal coordinate for $\mathrm{CHBrClF}$ [109] using the deMon program package

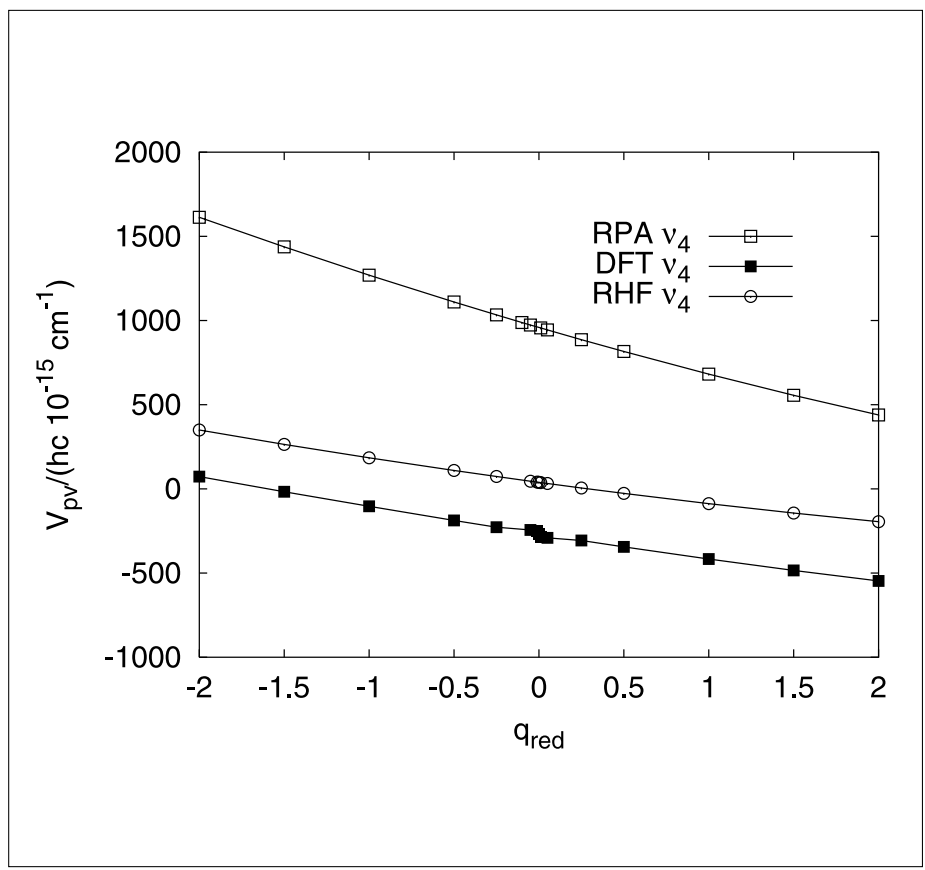

Fig. 3. DFT calculation (LSD approximation and VWN parametrization of the correlation functional) as a function of the CF-stretching reduced normal coordinate for CHBrCIF [109] as compared to restricted HF and RPA calculations 
Table 3. Molecules for which the influence of parity violation on molecular properties relevant for the infrared and microwave spectral ranges has been examined computationally: standard heat of reaction for stereomutation at $T=0 \mathrm{~K}, \Delta_{\mathrm{pv}} H_{0}^{0}$; relative (vibrational) frequency shift, $\Delta_{\mathrm{pv}} v_{i} / v_{i}(i=1, \ldots 3 N-6)$, where $N$ is the number of atoms); relative change of the rotational constants $A, B, C, \Delta_{\mathrm{pv}} X / X,(X=A$, $B, C$ ); equilibrium constant, $K_{\mathrm{p}}$; heat capacity at constant volume, $C_{V}$.

\begin{tabular}{|c|c|c|c|}
\hline Molecule & Year & Reference(s) & Properties \\
\hline \multirow{3}{*}{$\mathrm{CHBrClF}$} & $1999^{a} / 2000$ & {$[57]$} & $\begin{array}{l}\Delta_{\mathrm{pv}} H_{0}^{0}, \Delta_{\mathrm{pv}} X / X,(X=A, B, C) \\
\Delta_{\mathrm{pv}} v_{i} / v_{i},(i=1, \ldots, 9)\end{array}$ \\
\hline & 2000 & {$[61]$} & $\Delta_{\mathrm{pv}} v / v$ (three stretching modes) \\
\hline & 2000 & {$[102]$} & $\Delta_{\mathrm{pv}} v_{i} / v_{i},(i=1, \ldots, 9)$ \\
\hline \multirow{4}{*}{ CDBrCIF } & 2000 & {$[58]$} & $\Delta_{\mathrm{pv}} X / X,(X=A, B, C)$ \\
\hline & & & $\Delta_{\mathrm{pv}} v / v,(i=1, \ldots, 9)$ (and overtones) \\
\hline & 2001 & {$[59]$} & $\Delta_{\mathrm{pv}} H_{0}^{0}, K_{p,} C_{V}$ \\
\hline & 2003 & {$[60]$} & $\Delta_{\mathrm{pv}} v_{i} / v_{i}(4$-dimensional, coupled $)$ \\
\hline $\begin{array}{l}\text { CFXYZ (X, Y, Z = H, Br, } \\
\text { Cl, I) }\end{array}$ & 2002 & [103] & $\Delta_{\mathrm{pv}} v / v(\mathrm{CF}-\mathrm{stretch})$ \\
\hline F-oxirane & 2001 & [104] & $\begin{array}{l}\Delta_{\mathrm{pv}} H_{0}^{0} K_{p} \\
\Delta_{\mathrm{pv}} v / v(i=1, \ldots, 9)\end{array}$ \\
\hline $\mathrm{BiHFBr}, \mathrm{BiHFI}$ & 2003 & [105] & $\Delta_{\mathrm{pv}} v_{i} / v_{i}$ (four modes) \\
\hline $\begin{array}{l}\mathrm{PH}_{3} \mathrm{AuCHFCl}, \\
\mathrm{ClHgCHFCl}\end{array}$ & 2003 & [106] & $\Delta_{\mathrm{pv}} v / v(\mathrm{CF}-\mathrm{stretch})$ \\
\hline Camphor & 2004 & {$[107]$} & $\Delta_{\mathrm{pv}} v / v$ (one mode) \\
\hline $\mathrm{CBrClF}^{+}, \mathrm{CBrClF}^{-}$ & 2004 & {$[69]$} & $\Delta_{\mathrm{pv}} H_{0, \Delta_{\mathrm{pv}}}^{0} v_{i} / v_{i},(i=1, \ldots, 6)$ \\
\hline PHBrF, AsHBrF & 2004 & {$[108]$} & $\Delta_{\mathrm{pv}} v_{i} / v_{i},(i=1, \ldots, 6)$ \\
\hline
\end{tabular}

aPresented at the $37^{\text {th }}$ IUPAC Meeting, Berlin (August 1999)

accurate than high quality ab initio methods and thus probably less useful for the analysis of accurate spectroscopic results in the future.

\subsection{Relative Importance of Corrections Due to Relativistic and Vibrational Effects}

There is no doubt that for highly charged nuclei in the lowest rows of the periodic system only relativistic calculations can be accurate, while for the first rows the semi-relativistic approximation should be adequate. Relativistic corrections should be considered in the intermediate range and might be compared to other corrections, for instance from multidimensional vibrational ground state wavefunctions. This question has been investigated for the 'intermediate' molecule $\mathrm{CDBrClF}$ and we provide a brief summary freedom) which is also much more economic than the numerical integration of the Schrödinger equation (using 5000 points for one dimension) as employed in [93]. However, as has been discussed in great detail [60], Taylor expansions are of rather limited use in variational (ro)vibrational calculations due to difficulties with respect to convergence and the frequent occurrence of resonances. Accurate approaches for such calculations have been discussed and carried out in [60].

A possible extension of the calculations of vibrational effects in the future might be the use of density functional techniques (Section 4.4) combined with Car-Parrinello simulations, which would render very large molecular systems accessible for such vibrational effects, although at lower accuracy.

\subsection{A New Isotope Effect}

The weak nuclear interaction leads to a fundamentally new isotope effect, which normally is negligibly small, but becomes important for molecules that are chiral just by isotopic substitution such as $\mathrm{PF}^{35} \mathrm{Cl}^{37} \mathrm{Cl}$. This new isotope effect was investigated recently with this and related examples [112]. Molecular isotope effects are due to the following basic origins:

i) Mass differences of the isotopes, quite commonly [14].

ii) Different spins of the isotopes [113].

iii) Different symmetry properties of the molecular wavefunction for different isotopomers, which arise irrespective of differences of mass and spin, for instance nuclear isomers of almost the same mass and sometimes the same spin [114].

iv) The different electroweak charge $Q_{a}$ of different isotopes following Eqn. (7).

While the first three of these have been known for some time, the last has been considered only recently by quantitative calculations [112]. It turns out that the effect leads to parity violating energy differences of enantiomers in isotopically chiral molecules. With moderately heavy isotopes such as ${ }^{35} \mathrm{Cl} /{ }^{37} \mathrm{Cl}$ pairs, these are not very much smaller than for usual chiral molecules. This renders such molecules also useful for experiments and may have consequences for spectroscopy and chemistry of such isotopically chiral molecules [112].

\section{Conclusions}

From the short review of the theory and a few examples of results it should be clear that the theoretical work starting about a decade ago has provided the essential breakthrough that was necessary towards a formulation and realization of an accurate theory of molecular parity violation [35]. Independent confirmation of our results by several other research groups renders it 
Table 4. Selection of relative harmonic (har.) and anharmonic (anh.) frequency shifts $\Delta_{\mathrm{pv}} \tilde{\mathrm{v}} / \tilde{\mathrm{v}}$ (in 10 ${ }^{-19}$ ) [60] obtained with different ab initio methods (RPA: MCLR Random Phase Approximation, CASSCF: Complete Active Space Self Consistent Field approach (we used 12 electrons distributed in 12 orbitals [12,12], see [58]), DHF: (relativistic) Dirac-Hartree-Fock approach) for some selected stretching and bending modes. The various calculations consider anharmonicity effects without coupling (1D) and with coupling (4D, Model C) for $(R)$ - $\mathrm{CHBrCIF}$ and its isotopomer $(R)$-CDBrCIF as indicated in the fourth top line.

$$
\Delta_{\mathrm{pv}} \tilde{v} /\left(10^{-19} \tilde{\mathrm{v}}\right)
$$

\begin{tabular}{|c|c|c|c|c|c|c|c|c|}
\hline & \multicolumn{4}{|c|}{$\mathrm{CHBrCIF}$} & \multicolumn{3}{|c|}{ CDBrCIF } & \\
\hline & \multicolumn{2}{|c|}{ MCLR-RPA [55-58] } & \multirow{2}{*}{$\begin{array}{c}\text { CASSCF [58] } \\
\text { 1D anh. }\end{array}$} & \multirow{2}{*}{$\begin{array}{l}\text { DHF [61] } \\
\text { 1D anh. }\end{array}$} & \multicolumn{2}{|c|}{ RPA [59] } & \multirow{2}{*}{$\begin{array}{l}\text { RPA [60] } \\
\text { 4D anh. }\end{array}$} & \\
\hline Mode & 1D har. & 1D anh. & & & 1D har. & 1D anh. & & Mode \\
\hline $\mathrm{v}_{4}$, CF stretch & 305.9 & -805.9 & -663.8 & -623.0 & 371. & -1351.3 & -2144.4 & $v_{2}$, CF stretch \\
\hline $\mathrm{v}_{5}, \mathrm{CCl}$ stretch & -1499.6 & -2499.5 & & -3028.5 & -1445.1 & -3134.0 & & $\mathrm{v}_{5}, \mathrm{CCl}$ stretch \\
\hline $\mathrm{v}_{6}, \mathrm{CBr}$ stretch & 593.4 & 952.9 & & 552.0 & 723.5 & 1167.3 & & $\mathrm{v}_{6}, \mathrm{CBr}$ stretch \\
\hline $\mathrm{v}_{8}, \mathrm{BrCF}$ bend & -293.8 & -3360.7 & & & -289.3 & -2814.9 & & $v_{8}, B r C F$ bend \\
\hline$\Delta_{\mathrm{pv}} E /\left(h c 10^{-12} \mathrm{~cm}^{-1}\right)$ & $1.92^{\mathrm{a}}$ & $1.90^{\mathrm{b}}$ & $1.89^{b}$ & 2.33 & $1.92^{\mathrm{a}}$ & $1.90^{\mathrm{b}}$ & $1.96^{a}, 1.76^{b}$ & \\
\hline
\end{tabular}

likely that the current results are, indeed, accurate. This will be the basis for further refinement of theory and its application to the planning and analysis of experiments which are currently being pursued. There is no doubt that activity in this formerly very small research field has increased and will increase further in the future. The fruits being harvested already and to be harvested still in the future, concern a better understanding of the foundation of molecular chirality, biomolecular homochirality and perhaps even the Standard Model of Elementary Particle Physics.

\section{Acknowledgement}

We gratefully acknowledge collaboration with M. Casida, C. Jamorski and D. Salahub (Université de Montréal) on the density functional aspect of our work. Discussions with S. Albert, R. Berger, M. Gottselig, H. Hollenstein, D. Luckhaus, A. Sieben, and M. Willeke are gratefully acknowledged. Our work is supported financially by the Swiss National Science Foundation, the Alliance for Global Sustainability (AGS), and ETH Zürich (including RZETH, CSCS Manno and C4).

Received: June 10, 2005

[1] W. Heisenberg, Z. Phys. 1925, 33, 879.

[2] M. Born, P. Jordan, Z. Phys. 1925, 34, 858.

[3] M. Born, W. Heisenberg, P. Jordan, Z. Phys. 1926, 35, 557.

[4] E. Schrödinger, Ann. Phys. IV. Folge 1926, 81, 109.

[5] P. Dirac, Proc. Roy. Soc. (London) 1929, A123, 714.
[6] W. Heitler, F. London, Z. Physik 1927, 44, 455.

[7] F. Hund, Z. Physik 1926, 40, 742

[8] R. Mulliken, J. Chem. Phys. 1933, 1, 492.

[9] L. Pauling, 'The nature of the chemical bond and the structure of molecules and crystals: An introduction to modern structural chemistry', Cornell University Press, Ithaca, NY, 1960, $3^{\text {rd }}$ ed.

[10] F. Hund, Z. Physik 1927, 43, 788.

[11] F. Hund, Z. Physik 1931, 73, 1.

[12] J.A. Pople, Angew. Chem. Intl. Ed. (Engl.) 1999, 38, 1894.

[13] W. Kohn, Rev. Mod. Phys. 1999, 71, 1253.

[14] M. Quack, Annu. Rev. Phys. Chem. 1990, $41,839$.

[15] M. Quack, 'Molecular femtosecond quantum dynamics between less than yoctoseconds and more than days: Experiment and theory', chapter 27 in 'Femtosecond Chemistry', Eds. J. Manz, L. Woeste, Verlag Chemie, Weinheim, 1995, p. 781818.

[16] V. Alezra, G. Bernardelli, C. Corminbœuf, U. Frey, E.P. Kundig, S.E. Merbach, C.M. Saudan, F. Viton, J. Weber, J. Am. Chem. Soc. 2004, 126, 4843.

[17] C.A. Daul, Chimia 2004, 58, 316.

[18] R. Car, M. Parrinello, Phys. Rev. Lett. 1985, 55, 2471.

[19] W.F. van Gunsteren, H.J.C. Berendsen, $A n-$ gew. Chem. Intl. Ed. (Engl.) 1990, 29, 992.

[20] P. Carloni, U. Roethlisberger, M. Parrinello, Acc. Chem. Res. 2002, 35, 455.

[21] F. Mariotti, M. Quack, M. Willeke, J. Stohner, Chimia 2004, 58, 263.

[22] T.D. Lee, C.N. Yang, Phys. Rev. 1956, 104, 254.
[23] C.S. Wu, E. Ambler, R.W. Hayward, D.D. Hoppes, R.P. Hudson, Phys. Rev. 1957, 105, 1413.

[24] R.L. Garwin, L.M. Lederman, M. Weinrich, Phys. Rev. 1957, 105, 1415.

[25] J.I. Friedman, V.L. Telegdi, Phys. Rev. 1957, 105, 1681.

[26] M.L. Goldhaber, L. Grodzins, A. Sunyar, Phys. Rev. 1958, 109, 1015.

[27] B. Zel'dovich, Sov. Phys. JETP 1959, 9, 682.

[28] D.E. Groom, M. Aguilar-Benitez, C. Amsler, R.M. Barnett, P.R. Burchat, C.D. Carone, C. Caso, G. Conforto, O. Dahl, M. Doser, S. Eidelman, J. Feng, L. Gibbons, M. Goodman, C. Grab, A. Gurtu, K. Hagiwara, K. Hayes, J. Hernández, K. Hikasa, K. Honscheid, C. Kolda, M. Mangano, A. Manohar, A. Masoni, K. Mönig, H. Murayama, K. Nakamura, S. Navas, K. Olive, L. Pape, A. Piepke, M. Roos, M. Tanabashi, N. Törnquist, T. Trippe, P. Vogel, C. Wohl, R. Workman, W.-M. Yao, Eur. Phys. J. 2000, C 15,1 .

[29] H. Schopper, Nova Acta Leopoldina 1999, NF81, 109.

[30] M.J.G. Veltman, Rev. Mod. Phys. 2000, 72, 341.

[31] G. 't Hooft, Rev. Mod. Phys. 2000, 72, 333.

[32] Y. Yamagata, J. Theoret. Biol. 1966, 11, 495.

[33] D. Rein, J. Mol. Evol. 1974, 4, 15.

[34] M. Quack, Angew. Chem. Int. Ed. Engl. 1989, 28, 571.

[35] A. Bakasov, T.K. Ha, M. Quack, in 'Proc. of the 4 Trieste Conference (1995), Chemical Evolution: Physics of the Origin and 
[36] A. Bakasov, T.K. Ha, M. Quack, J. Chem. Phys. 1998, 109, 7263.

[37] A. Bakasov, T.K. Ha, M. Quack, J. Chem. Phys. 1999, 110, 6081.

[38] R. Berger, M. Quack, ChemPhysChem. 2000, 1,57 .

[39] R. Berger, M. Quack, G. Tschumper, Helv Chim. Acta 2000, 83, 1919.

[40] M. Quack, Angew. Chem. Intl. Ed. (Engl.) 2002, 41, 4618 .

[41] W.A. Bonner, Topics in Stereochemistry 1988, $18,1$.

[42] W.A. Bonner, CHIRALITY 2000, 12, 114.

[43] P. Frank, W.A. Bonner, R. Zare,in 'Chemistry for the $21^{\text {st }}$ century', Ed. E. Keinan, I. Schechter, Wiley-VCH, Weinheim, 2000, pp. 175-208.

[44] D.W. Rein, R.A. Hegstrom, P.G.H. Sandars, Phys. Lett. 1979, A71, 499.

[45] R.A. Hegstrom, D.W. Rein, P.G.H. Sandars, J. Chem. Phys. 1980, 73, 2329.

[46] S.F. Mason, G.E. Tranter, Mol. Phys. 1984, 53,1091

[47] G.E. Tranter, Mol. Phys. 1985, 56, 825.

[48] One exception may be the Italian group which published their first contribution in this field in 1997. Lazzeretti and Zanasi stated in a letter to us in 1999 that they were unaware of our earlier work; private communication (letter to M. Quack in 1999).

[49] O. Kompanets, A. Kukudzhanov, V. Letokhov, L. Gervits, Opt. Commun. 1976, 19, 414.

[50] E. Arimondo, P. Glorieux, T. Oka, Opt. Commun. 1977, 23, 369.

[51] C. Daussy, T. Marrel, A. Amy-Klein, C. Nguyen, C. Bordé, C. Chardonnet, Phys. Rev. Lett. 1999, 83, 1554.

[52] M. Ziskind, C. Daussy, T. Marrel, C. Chardonnet, Eur. Phys. J. 2002, D 20, 219.

[53] A. Bauder, A. Beil, D. Luckhaus, F. Müller, M. Quack, J. Chem. Phys. 1997, 106, 7558.

[54] A. Lahamer, S. Mahurin, R. Compton, D. House, J. Laerdahl, M. Lein, P. Schwerdtfeger, Phys. Rev. Lett. 2000, 85, 4470.

[55] J. Stohner, A. Beil, H. Hollenstein, O. Monti, M. Quack, in '37 IUPAC Congress and 27 GDCh Meeting, Berlin, Germany, August 14-19, 1999, Frontiers in Chemistry: Molecular Basis of the Life Sciences', ISBN 3-924763-82-8, p. 525.

[56] M. Quack, J. Stohner, in 'SASP 2000, Proc. of the XII Symposium on Atomic and Surface Physics and related topics, January 30 - February 5, 2000', Eds. Davide Bassi, Paolo Tosi, Folgaria (Universita di Trento) Italy 2000, $P R-11$, p. 1-4.

[57] M. Quack, J. Stohner, Phys. Rev. Lett. 2000, 84, 3807.

[58] M. Quack, J. Stohner, Z. Physik. Chemie 2000, 214, 675.

[59] M. Quack, J. Stohner, CHIRALITY 2001, 13, 745 .

[60] M. Quack, J. Stohner, J. Chem. Phys. 2003 , $119,11228$.

[61] J.K. Laerdahl, P. Schwerdtfeger, H.M. Quiney, Phys. Rev. Lett. 2000, 84, 3811.

[62] A. Soncini, A. Ligabue, P. Lazzeretti, R. Zanasi, Phys. Rev. E 2000, 62, 8395.
[63] M. Quack, Chem. Phys. Lett. 1986, 132, 147.

[64] R. Harris, L. Stodolski, Phys. Lett. 1978 $B 78,313$.

[65] R. Harris, L. Stodolski, J. Chem. Phys. 1981, 74, 2145.

[66] M. Gottselig, D. Luckhaus, M. Quack, J. Stohner, M. Willeke, Helv. Chim. Acta 2001, 84, 1846.

[67] M. Gottselig, M. Quack, M. Willeke, Isr. J. Chem. 2003, 43, 353.

[68] M. Gottselig, M. Quack, J. Stohner, M. Willeke, Int. J. Mass Spectrometry 2004 233, 373.

[69] J. Stohner, Int. J. Mass Spectrometry 2004, 233,385

[70] M. Gaillard, P. Grannis, F. Sciulli, Rev. Mod. Phys. 1999, 71, S96.

[71] D.H. Perkins, 'Introduction to High Energy Physics', Cambridge University Press, Cambridge, 2000, $4^{\text {th }}$ ed.

[72] T. Cvitaš, J. Frey, B. Holmström, K. Kuchitsu, R. Marquardt, I. Mills, F. Pavese, M. Quack, J. Stohner, H.L. Strauss, M. Takami, A.J. Thor, 'Quantities, Units and Symbols in Physical Chemistry', RSC, Oxford; in preparation, $\mathbf{2 0 0 5}$

[73] H. Kiyonaga, K. Morihashi, O. Kikuchi, J. Chem. Phys. 1998, 108, 2041.

[74] A.L. Barra, J.B. Robert, L. Wiesenfeld, Europhys. Lett. 1988, 5, 217.

[75] R. Berger, in 'Relativistic Electronic Structure Theory', Part 2, Ed. P. Schwerdtfeger Chapter 4, Elsevier, Amsterdam, 2004, pp. 188-288.

[76] I.B. Khriplovich, 'Parity Nonconservation in Atomic Phenomena', Gordon and Breach, Philadelphia, 1991

[77] J.K. Laerdahl, P. Schwerdtfeger, Phys. Rev. 1999, A60, 4439.

[78] A.L. Barra, J.B. Robert, Mol. Phys. 1996, $88,875$.

[79] R. Berger, M. Quack, J. Chem. Phys. 2000, 112,3148

[80] M. Martinez, R. Miquel, L. Rolandi, R. Tenchini, Rev. Mod. Phys. 1999, 71, 575.

[81] G. Laubender, R. Berger, ChemPhysChem 2003, 4, 395

[82] A. Soncini, F. Faglioni, P. Lazzeretti, Phys. Rev. A 2003, 68, 033402.

[83] S. Blundell, J. Sapirstein, W. Johnson, Phys Rev. D 1992, 45, 1602.

[84] H. Quiney, H. Skaane, I. Grant, Adv. Quant Chem. 1999, 32, 1

[85] S.F. Mason, G.E. Tranter, Chem. Phys. Lett. 1983, 94, 34 .

[86] A second computer program had to be developed completely independently in 1996 (JS). It was much more efficient by exploiting fast matrix multiplication techniques for the MO transformation and it was easier to use than the original version devised by A. Bakasov.

[87] J. Linderberg, Y. Öhrn, 'Propagators in Quantum Chemistry', Academic Press, London, 1973.

[88] O. Vahtras, H. Agren, P. Jorgensen, J. Jen- sen, T. Helgaker, J. Olsen, J. Chem. Phys. 1992, 96, 2118.

[89] P. Lazzeretti, R. Zanasi, Chem. Phys. Lett. 1997, 279, 349.

[90] J. Thyssen, J. Laerdahl, P. Schwerdtfeger, Phys. Rev. Lett. 2000, 85, 3105.

[91] R. Berger, N. Langermann, C. van Wüllen, Phys. Rev. 2005, A71, 042105.

[92] R. Berger, C. van Wüllen, J. Chem. Phys. 2005, 122, 134316.

[93] P. Schwerdtfeger, T. Saue, J.N.P. van Stralen, L. Visscher, Phys. Rev. A 2005, 71 , 012103.

[94] A. Bakasov, M. Quack, Chem. Phys. Lett. 1999, 303, 547, A. Bakasov, R. Berger, T. K. Ha, M. Quack, Int. J. Quant. Chem. 2004, 98, 393.

[95] G.E. Tranter, Chem. Phys. Lett. 1985, 120, 93.

[96] O. Kikuchi, H. Wang, Bull. Chem. Soc. Japan 1990, 63, 2751.

[97] R. Janoschek, in 'Chirality', Springer-Verlag, Berlin, 1991, p. 18.

[98] A.J. MacDermott, in 'Chirality in Natural and Applied Science', Eds. W.J. Lough, I.W. Wainer, Blackwell, Oxford, 2002, pp. 23-52.

[99] J.K. Laerdahl, R. Wesendrup, P. Schwerdtfeger, ChemPhysChem 2000, 1, 60 .

[100] T. Watanabe, K. Morihashi, O. Takahashi, T. Kitayama, T. Yagi, O. Kikuchi, J. Mol. Struct. (Theochem) 2004, 671, 119.

[101] M. Quack, Nova Acta Leopoldina 1999, NF 81, 137.

[102] R. Viglione, R. Zanasi, P. Lazzeretti, A. Ligabue, Phys. Rev. A 2000, 62, 052516.

[103] P. Schwerdtfeger, J. Laerdahl, C. Chardonnet, Phys. Rev. A 2002, 65, 042508

[104] R. Berger, M. Quack, J. Stohner, Angew. Chem. Intl. Ed. (Engl.) 2001, 40, 1667.

[105] F. Faglioni, P. Lazzeretti, Phys. Rev. 2003 A67, 032101.

[106] R. Bast, P. Schwerdtfeger, Phys. Rev. Lett. 2003, 91, 023001.

[107] P. Schwerdtfeger, A. Kuhn, R. Bast, J. Laerdahl, F. Faglione, P. Lazzeretti, Chem. Phys. Lett. 2004, 383, 496.

[108] R. Viglione, J. Chem. Phys. 2004, 121, 9959.

[109] J. Stohner, M.E. Casida, D.R. Salahub, M. Quack, unpublished 2000.

[110] M.E. Casida, C. Daul, A. Goursot, A. Koester, L. Pettersson, E. Proynov, A. St-Amant, D.R. Salahub, H. Duarte, N. Godbout, J. Guan, C. Jamorski, M. Lebœuf, V. Malkin, O. Malkina, F. Sim, A. Vela, deMon KS Ver sion 3.41996 .

[111] A. Hennum, T. Helgaker, W. Klopper, Chem. Phys. Lett. 2002, 354, 274.

[112] R. Berger, G. Laubender, M. Quack, A. Sieben, J. Stohner, M. Willeke, Angew. Chem. Intl. Ed. (Engl.) 2005, 44, 3623.

[113] R.R. Ernst, Angew. Chem. Intl. Ed. (Engl.) 1992, 31, 805 .

[114] M. Quack, Mol. Phys. 1977, 34, 477. 